\title{
Well-posedness of domain integral equations for a dielectric object in homogeneous background
}

\author{
M. C. van Beurden · S. J. L. van Eijndhoven
}

Received: 29 March 2007 / Accepted: 11 February 2008 / Published online: 6 March 2008

(C) The Author(s) 2008

\begin{abstract}
An analysis of the mapping properties of three commonly used domain integro-differential operators for electromagnetic scattering by an inhomogeneous dielectric object embedded in a homogeneous background is presented in the Laplace domain. The corresponding three integro-differential equations are shown to be equivalent and well-posed under finite-energy conditions. The analysis allows for non-smooth changes, including edges and corners, in the dielectric properties. The results are obtained via the Riesz-Fredholm theory, in combination with the Helmholtz decomposition and the Sobolev embedding theorem.
\end{abstract}

Keywords Dielectric objects $\cdot$ Electromagnetic scattering $\cdot$ Integral equations $\cdot$ Well-posedness

\section{Introduction}

The study of electromagnetic scattering by strongly inhomogeneous objects has many biological and geophysical applications ranging from hyperthermia cancer therapy, to determination of specific absorption rates (SAR) in the human head for product designs such as in GSM or UMTS applications, to exploration of the soil with respect to ores, to the detection of anti-personnel mines. In inverse scattering, one seeks to determine the material composition of an object in terms of its electromagnetic properties.

The electromagnetic scattering phenomena are usually studied in a numerical context, based either on partial differential equations or on domain integro-differential equations. One of the main advantages of the latter is that, in principle, they can be restricted to the domain of the scattering object itself, and the radiation conditions at infinity are automatically satisfied, as opposed to finite-element and finite-difference techniques, which originate from partial differential equations. On the other hand, without special measures, the computational complexity of discretized versions of integro-differential equations is much higher compared to finite-element and finite-difference methods for corresponding partial differential equations; the former gives rise to dense matrices, while the latter give highly sparse matrices. In terms of the number of unknowns, the discretized integro-differential equations are usually more

M. C. van Beurden ( $\varangle)$

Department of Electrical Engineering, Eindhoven University of Technology, Eindhoven, The Netherlands

e-mail: m.c.v.beurden@tue.nl

S. J. L. van Eijndhoven

Department of Mathematics and Computer Science, Eindhoven University of Technology, Eindhoven, The Netherlands 
economical; in finite-element and finite-difference contexts, extra space is required to deal with artificial boundary conditions to take into account approximated radiation conditions.

For integro-differential equations, several methods have been proposed to overcome the complexity problem, such as the CGFFT [1-3], which is based on FFTs on a structured grid, and the multilevel fast multipole algorithm (MLFMA) [4]. All aim at the construction of an efficient matrix-vector product and employ iterative techniques to solve the set of linear equations. Nevertheless, since the scattering object is inhomogeneous, a full three-dimensional mesh is needed to arrive at a discretization of the pertaining integro-differential equation, which leads to a large number of unknowns and large sets of linear equations. Under certain conditions, the convergence of the aforementioned iterative techniques tends to stall, e.g., for large differences in the permittivity between the object and its surrounding background material, or for negative contrasts, i.e., the case where the permittivity of the background is higher than the one of the object itself. Such observations raise the question as to whether the integro-differential equations are properly discretized and what else might be the cause of the stalling of the convergence. Can this convergence problem be overcome by preconditioning techniques and how should this preconditioner be constructed?

As a first step towards answering these questions, we present an analysis of the mapping properties of three commonly used integro-differential equations for scattering by an inhomogeneous dielectric object embedded in a homogeneous space, in the Laplace domain. The next step is then to adapt existing discretization procedures to arrive at a numerical scheme that accounts for these mapping properties or identify parts of the integro-differential operator that require special treatment in the form of preconditioning.

The domain integro-differential equation for the electric field for a dielectric object in a homogeneous background was analyzed by Müller [5], under the assumption that the permittivity functions are continuously differentiable with respect to the spatial variables. Although this model is often encountered in real life, it is not so frequently encountered in numerical models. For permittivity functions with discontinuities, sharp edges, or corners, the analysis of Müller [5] breaks down. Recently, the same domain integro-differential equation was analyzed by means of the Banach contraction principle in [6-9] under finite-energy conditions. In this analysis, no assumptions are made on the smoothness of the permittivity functions, and specific details regarding the background medium are not required, although both the object and the background need to be lossy. In the analysis presented here, the dielectric object can have spatial discontinuities in its dielectric material and the boundaries on which the discontinuities occur are allowed to have sharp edges and corners. Further, we allow for lossy and lossless dielectric properties for both the object and the homogeneous space in which it is embedded, and the time-dependence can be purely harmonic. Our mathematical analysis is based on the Riesz-Fredholm theory, in combination with the Helmholtz decomposition and the Sobolev embedding theorem. To deal with corners and edges, we restrict the analysis to the case where the electromagnetic field carries a locally finite energy.

\section{Formulation of the domain integro-differential equations}

A dielectric object occupying the bounded domain $\mathcal{D}$ in 3-D Euclidean space, with boundary $\partial \mathcal{D}$, is described by a permittivity function $\varepsilon(\boldsymbol{r}, \tau)$, where $\boldsymbol{r}$ is the (Cartesian) position vector, and $\tau$ is the time lag between the force action and the reaction of the medium to model dispersion. The permittivity function is, in general, a $3 \times 3$ tensor. The configuration is depicted in Fig. 1 . Outside $\overline{\mathcal{D}}=\mathcal{D} \cup \partial \mathcal{D}$, the permittivity function equals the permittivity $\varepsilon_{0}$ of free space, although the extension to another homogeneous background is straightforward. We assume that the permeability equals $\mu_{0}$ everywhere. We apply a Laplace transform with respect to time to Maxwell's equations, according to

$$
\boldsymbol{F}(s)=\int_{0}^{\infty} \mathcal{F}(t) \exp (-s t) \mathrm{d} t, \quad \mathfrak{R e}\{s\} \geq 0 .
$$

In most cases, we let the real part of the Laplace parameter $s$ become zero, which should be understood as

$$
s=\lim _{\delta \downarrow 0} \delta+\mathrm{j} \omega, \quad(\mathrm{j}=\sqrt{-1}),
$$


Fig. 1 A dielectric object with incident field

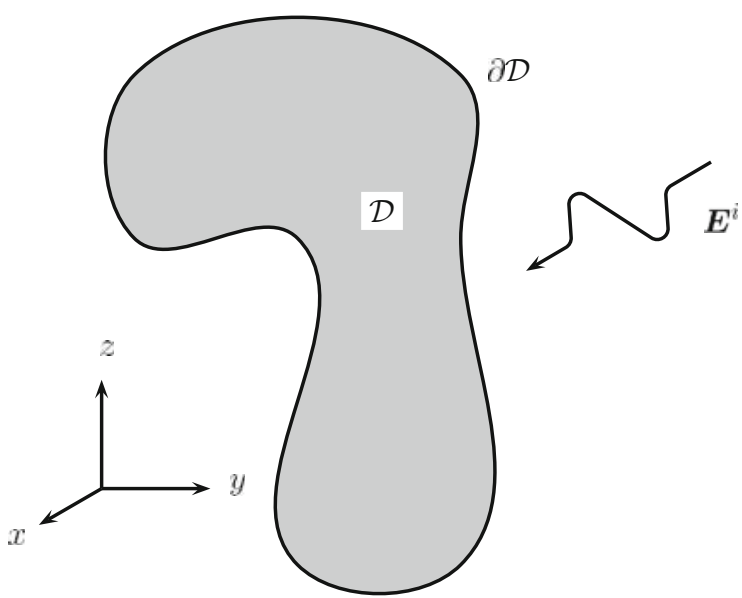

assuming that $\mathcal{F}(t)$ is integrable with respect to $t$, and $\omega \geq 0$ is the radial frequency. Maxwell's equations can now be written as

$\nabla \times \boldsymbol{H}(\boldsymbol{r}, s)=s \varepsilon(\boldsymbol{r}, s) \boldsymbol{E}(\boldsymbol{r}, s)+\boldsymbol{J}_{0}(\boldsymbol{r}, s), \quad \nabla \times \boldsymbol{E}(\boldsymbol{r}, s)=-s \mu_{0} \boldsymbol{H}(\boldsymbol{r}, s)-\boldsymbol{K}_{0}(\boldsymbol{r}, s)$,

where $\boldsymbol{E}$ and $\boldsymbol{H}$ denote the electric and magnetic fields, and $\boldsymbol{J}_{0}$ and $\boldsymbol{K}_{0}$ denote the primary electric and magnetic sources. Further, we have assumed that the permittivity function also takes into account possible Ohmic losses. Subsequently, we write the electric and magnetic field as the sum of the incident field and the scattered field, indicated with a superscript $i$ and $s$, respectively, i.e.,

$\boldsymbol{E}=\boldsymbol{E}^{i}+\boldsymbol{E}^{s}, \quad \boldsymbol{H}=\boldsymbol{H}^{i}+\boldsymbol{H}^{s}$.

The incident fields satisfy Maxwell's equations in the absence of the dielectric object and in the presence of the primary sources. Consequently, the scattered fields satisfy

$\nabla \times \boldsymbol{H}^{s}=s \varepsilon_{0} \boldsymbol{E}^{s}+s \varepsilon_{0} \chi \boldsymbol{E}=s \varepsilon_{0} \boldsymbol{E}^{s}+\boldsymbol{J}^{c}, \quad \nabla \times \boldsymbol{E}^{s}=-s \mu_{0} \boldsymbol{H}^{s}$.

We have introduced $\chi(\boldsymbol{r}, s)$, which is a $3 \times 3$ tensor with $\chi_{k, \ell}(\boldsymbol{r}, s)=\left(\varepsilon_{k, \ell}(\boldsymbol{r}, s)-\varepsilon_{0} \delta_{k, \ell}\right) / \varepsilon_{0}$. Further, we have defined the contrast-current density $\boldsymbol{J}^{c}=s \varepsilon_{0} \chi \boldsymbol{E}$, which has support $\overline{\mathcal{D}}$. In the absence of a primary polarization field, another useful definition of the contrast-current density can be obtained from the relation $\boldsymbol{D}(\boldsymbol{r}, s)=\varepsilon(\boldsymbol{r}, s) \boldsymbol{E}(\boldsymbol{r}, s)$, where $\boldsymbol{D}$ is the generalized electric-flux density. This relation yields

$\boldsymbol{J}^{c}=s \varepsilon_{0} \chi \varepsilon^{-1} \boldsymbol{D}$,

under the assumption that the permittivity tensor is point-wise invertible.

The scattered electric and magnetic field can now be expressed in terms of a vector potential $\boldsymbol{A}$ of $\boldsymbol{H}^{s}$ as [10, Chap. 26]

$\boldsymbol{E}^{s}=\frac{1}{s \varepsilon_{0}}\left(\nabla \nabla \cdot \boldsymbol{A}-s^{2} \varepsilon_{0} \mu_{0} \boldsymbol{A}\right), \quad \boldsymbol{H}^{s}=\nabla \times \boldsymbol{A}$.

For continuously differentiable $\boldsymbol{J}^{c}$ with compact support $\mathcal{D}$ in $\mathbb{R}^{3}$, the vector potential is given by

$\boldsymbol{A}(\boldsymbol{r})=\iiint_{\mathcal{D}} G\left(\boldsymbol{r}-\boldsymbol{r}^{\prime}\right) \boldsymbol{J}^{c}\left(\boldsymbol{r}^{\prime}\right) \mathrm{d} V^{\prime}$,

where the Lorenz gauge has been applied, the vector fields are described in Cartesian components,

$G(\boldsymbol{r})=\frac{\exp \left(-s|\boldsymbol{r}| / c_{0}\right)}{4 \pi|\boldsymbol{r}|}$

is the Green's function of the Helmholtz equation in free space, and $c_{0}$ is the velocity of light in free space. 
To arrive at a domain integro-differential equation for the contrast-current density, we employ Eq. (4a) resulting in the expression

$s \varepsilon_{0} \boldsymbol{E}^{i}=s \varepsilon_{0}\left(\boldsymbol{E}-\boldsymbol{E}^{s}\right)=s \varepsilon_{0} \boldsymbol{E}+\frac{s^{2}}{c_{0}^{2}} \boldsymbol{A}-\nabla \nabla \cdot \boldsymbol{A}$,

which, together with Eq. (8), is a domain integro-differential equation for $\boldsymbol{E}$ if we use the definition in Eq. (5a) for $\boldsymbol{J}^{c}$. Alternatively, we can obtain a domain integro-differential equation for $\boldsymbol{D}$, by using the constitutive relation between $\boldsymbol{E}$ and $\boldsymbol{D}$, which leads to

$s \varepsilon_{0} \boldsymbol{E}^{i}=s \varepsilon_{0} \varepsilon^{-1} \boldsymbol{D}+\frac{s^{2}}{c_{0}^{2}} \boldsymbol{A}-\nabla \nabla \cdot \boldsymbol{A}$.

By using relation (6), we see that $\boldsymbol{A}$ is a function of $\boldsymbol{D}$. Finally, we obtain a domain integro-differential equation for $\boldsymbol{J}^{c}$ by multiplying Eq. (10) by the contrast function $\chi$ and using the definition of $\boldsymbol{J}^{c}$, which results in

$s \varepsilon_{0} \chi \boldsymbol{E}^{i}=\boldsymbol{J}^{c}+\chi\left[\frac{s^{2}}{c_{0}^{2}} \boldsymbol{A}-\nabla \nabla \cdot \boldsymbol{A}\right]$,

where $\boldsymbol{A}$ is a function of $\boldsymbol{J}^{c}$ as shown in Eq. (8).

For future notational convenience, we introduce the following operators. The identity operator is denoted by I; $\mathrm{M}_{\chi}$ and $\mathrm{M}_{\mathcal{\varepsilon}^{-1}}$ are multiplication operators that multiply the matrix functions $\chi(\boldsymbol{r}, s)$ and $\varepsilon(\boldsymbol{r}, s)^{-1}$, and the operator $\mathrm{N}$ is defined in terms of the vector potential as

$\mathrm{N} \boldsymbol{J}^{c}=\frac{s^{2}}{c_{0}^{2}} \boldsymbol{A}(\boldsymbol{r})-\nabla \nabla \cdot \boldsymbol{A}(\boldsymbol{r})$,

where the vector potential is a function of the contrast current density $\boldsymbol{J}^{c}$ as defined in Eq. (8). The three integrodifferential equations of Eqs. (10), (11), and (12) are now denoted as

$$
\left(\mathrm{I}+\mathrm{NM}_{\chi}\right) \boldsymbol{E}=\boldsymbol{E}^{i}, \quad\left(\mathrm{I}+\mathrm{NM}_{\chi}\right) \mathrm{M}_{\varepsilon^{-1}} \boldsymbol{D}=\boldsymbol{E}^{i}, \quad \frac{1}{s \varepsilon_{0}}\left(\mathrm{I}+\mathrm{M}_{\chi} \mathrm{N}\right) \boldsymbol{J}^{c}=\mathrm{M}_{\chi} \boldsymbol{E}^{i}
$$

\section{Equivalence of the integro-differential equations}

The first issue to be resolved is the equivalence between the three integro-differential equations with respect to existence and uniqueness of the solution, i.e., if one of the equations admits a unique solution, then the other two also admit a unique solution. Below, we will explain why this is the case, under the assumption that the incident electric field is known everywhere.

\subsection{Existence}

First, let us consider the equivalence between Eqs. (14a) and (14c) for the total electric field and the contrast current density, respectively. Suppose that the total electric field $\boldsymbol{E}(\boldsymbol{r})$ has been solved in a volume containing $\mathcal{D}$ in its interior. Straightforward multiplication with the contrast function $\chi(\boldsymbol{r})$ immediately gives an expression for $\boldsymbol{J}^{c}(\boldsymbol{r})$. Since $\boldsymbol{E}(\boldsymbol{r})$ satisfies Eq. (14a), it is readily seen that the expression for $\boldsymbol{J}^{c}(\boldsymbol{r})$ satisfies Eq. (14c). Hence, $\boldsymbol{J}^{c}(\boldsymbol{r})$ can be directly obtained from the total electric field. The opposite situation is more complex because $\chi(\boldsymbol{r})$ is not necessarily invertible on $\mathcal{D}$ and certainly not invertible outside $\mathcal{D}$. To resolve this situation, we employ the scattered field representation given by Eq. (7a) in combination with Eq. (8). Since we have assumed that the incident electric field is known everywhere, we can reconstruct the total electric field $\boldsymbol{E}(\boldsymbol{r})$ from the sum of the incident field and the scattered field, which is expressed in terms of the contrast current density; in this way, the total electric field automatically satisfies Eq. (14a) by construction. Hence, we conclude that the domain integro-differential equations for the contrast current density and for the total electric field are equivalent. 
To settle the equivalence between Eqs. (14a) and (14c) for the total electric field and the contrast current density on the one hand and Eq. (14b) for generalized electric flux density $\boldsymbol{D}(\boldsymbol{r})$ on the other, we note that we can obtain $\boldsymbol{D}(\boldsymbol{r})$ from the total electric field by multiplication with the permittivity function $\varepsilon(\boldsymbol{r})$. In view of the equivalence between the contrast current density and the total electric field, as shown above, this step is sufficient to obtain $\boldsymbol{D}(\boldsymbol{r})$ from the contrast current density or from the total electric field. The reverse situation is settled by Eq. (6) and the equivalence between the total electric field and the contrast current density. Note that only the latter step requires the invertibility of the permittivity function at every point in space. This is not alarming, since the introduction of the generalized electric flux density already relies on this assumption and Eq. (14b) explicitly contains the inverse of the permittivity function. In this sense, the integro-differential equation for the generalized electric flux density is only conditionally equivalent to the other two integro-differential equations.

\subsection{Uniqueness}

To arrive at a uniqueness result for the integro-differential equations, we employ a uniqueness result for Maxwell's equations, i.e., we start from the uniqueness for the set of differential equations. The uniqueness result for Maxwell's equations, subject to the radiation conditions, for a dielectric object of finite size, i.e., the domain of the object is bounded in $\mathbb{R}^{3}$, with a finite number of sharp corners and edges in a homogeneous background was proven by Jones [11, Chap. 9]. To deal with corners and edges, Jones explicitly augmented the standard (Silver-Müller) radiation conditions with the conditions that the electric and magnetic fields are locally square-integrable. In the proof, the continuity of the tangential electric and magnetic fields at the interface between the homogeneous background and the dielectric object is used. Therefore, according to [11], we know that the following system has only the trivial solution:

$$
\left\{\begin{array}{l}
\nabla \times \boldsymbol{H}^{s}(\boldsymbol{r}, s)=s \varepsilon(\boldsymbol{r}, s) \boldsymbol{E}^{s}(\boldsymbol{r}, s) \\
\nabla \times \boldsymbol{E}^{s}(\boldsymbol{r}, s)=-s \mu_{0} \boldsymbol{H}^{s}(\boldsymbol{r}, s), \\
\text { radiationconditions } \wedge \boldsymbol{E}, \boldsymbol{H} \in L_{\mathrm{loc}}^{2}\left(\mathbb{R}^{3}\right)^{3},
\end{array}\right.
$$

where $\varepsilon$ equals $\varepsilon_{0}$ outside a bounded domain, and $L_{\text {loc }}^{2}\left(\mathbb{R}^{3}\right)^{3}$ is the space of three-dimensional vector functions which are locally square integrable. Let both $\boldsymbol{J}_{1}^{c}$ and $\boldsymbol{J}_{2}^{c}$ be solutions of Eq. (14c) with the same excitation. Then $\boldsymbol{J}_{3}^{c}=\boldsymbol{J}_{1}^{c}-\boldsymbol{J}_{2}^{c}$ is a solution of the homogeneous counterpart of Eq. (14c). The corresponding scattered fields $\boldsymbol{E}_{3}^{s}$ and $\boldsymbol{H}_{3}^{s}$ generated by this current density, and subject to the radiation conditions follow from Eqs. (7a), (7b), and (8). By definition of the current densities $\boldsymbol{J}_{1}^{c}=s \varepsilon_{0} \chi\left(\boldsymbol{E}^{i}+\boldsymbol{E}_{1}^{s}\right)$ and $\boldsymbol{J}_{2}^{c}=s \varepsilon_{0} \chi\left(\boldsymbol{E}^{i}+\boldsymbol{E}_{2}^{s}\right)$ we have $\boldsymbol{J}_{3}^{c}=s \varepsilon_{0} \chi\left(\boldsymbol{E}_{1}^{s}-\boldsymbol{E}_{2}^{s}\right)=s \varepsilon_{0} \chi \boldsymbol{E}_{3}^{s}$. Consequently, the fields $\boldsymbol{E}_{3}^{s}$ and $\boldsymbol{H}_{3}^{s}$ satisfy Eq. (15). We can conclude that $\boldsymbol{E}_{3}^{s}=\mathbf{0}$. Hence, $\boldsymbol{J}_{3}^{c}=\mathbf{0}$ and the integro-differential equation (14c) has a unique solution, provided that the solution is restricted to $L_{\text {loc }}^{2}\left(\mathbb{R}^{3}\right)^{3}$. The uniqueness for the other two domain integro-differential equations, i.e. Eqs. (14a) and (14b), can be established by virtually the same arguments.

\section{Decomposition and Fredholm properties of the operator}

In Sect. 3.1, we have shown that the three domain integro-differential equations are equivalent with respect to existence, i.e., if one equation admits a solution, then all equations do so. The existence of a solution itself has not yet been addressed. In the following, we demonstrate the existence of the solution and we show that it depends in a continuous way on the incident field. This, together with the uniqueness result of Sect. 3.2 and the equivalence, shows that all three integro-differential equations are well-posed, i.e., the operators have an inverse that is bounded.

In view of the equivalence between the three integro-differential operators, we can analyze any one of the three integro-differential operators. We choose to analyze the integro-differential equation for the contrast current density, i.e., Eq. (14c). The main motivation is that it is immediately clear that the domain of validity of the equation can be restricted to the support of the dielectric object, whereas for the other two equations, restriction of the domain is 
not straightforward. Further, the contrast current density does not require a priori smoothness conditions, such as tangential continuity for the electric field.

To analyse the mapping properties of the integro-differential operator I $+M_{\chi} N$ for $J^{c}$ defined in Eq. (14c), we invoke the Riesz theory for Fredholm operators of the second kind. The relevant part of the Riesz theory can be summarized as $[12,13]$ :

Theorem 1 Let $H_{1}$ and $H_{2}$ be Hilbert spaces, let $\mathrm{C}$ be a compact operator from $H_{1}$ to $H_{2}$ and let $\mathrm{T}$ be a bounded linear operator from $H_{1}$ to $H_{2}$ with $\mathrm{T}^{-1}$ bounded from $H_{2}$ to $H_{1}$. If $\mathrm{T}+\mathrm{C}: H_{1} \rightarrow H_{2}$ is one-to-one, then the inverse operator $(\mathrm{T}+\mathrm{C})^{-1}: H_{2} \rightarrow H_{1}$ exists and is bounded.

In terms of the operator $\mathrm{I}+\mathrm{M}_{\chi} \mathrm{N}$, the above theorem considers three items. First, we have to establish proper Hilbert spaces for the domain and range of the operator, i.e., we should identify $H_{1}$ and $H_{2}$. From the definition of the identity operator I, we have $H_{1} \subset H_{2}$. Further, in view of the conditions mentioned in Sect. 3.2 and the absence of smoothness conditions for $\varepsilon(\boldsymbol{r}, s)$, it is natural to identify both $H_{1}$ and $H_{2}$ with the Lebesgue space of square-integrable functions. Second, we have to show that the operator I $+M_{\chi} N$ is one-to-one. This effectively means that we have to show that the solution is unique, which was already shown in Sect. 3.2. Finally, we have to split the operator I $+\mathrm{M}_{\chi} \mathrm{N}$ such that I $+\mathrm{M}_{\chi} \mathrm{N}=\mathrm{T}+\mathrm{C}$, where $\mathrm{C}$ is compact from $H_{1}$ to $H_{2}$ and $\mathrm{T}$ has a bounded inverse on these Hilbert spaces. We will arrive at a suitable splitting of the operator by means of the Helmholtz decomposition.

\subsection{Conditions on the permittivity function}

First, we need to establish some properties of the permittivity function. Since we will use a Hilbert-space setting, it is natural to have conditions on the permittivity function in terms of functionals that have a meaning within such Hilbert spaces. Let us denote by $L^{2}(\mathcal{G})^{3}$ the space of three-dimensional Cartesian vector fields that are square-integrable on $\mathcal{G}$. For a bounded domain $\mathcal{G}$ that contains the dielectric object, we formulate the following conditions for the permittivity functions for fixed $s$ and all $f \in L^{2}(\mathcal{G})^{3}$,

$\alpha \iiint_{\mathcal{G}} \boldsymbol{f} \cdot \boldsymbol{f}^{*} \mathrm{~d} V \leq\left|\iiint_{\mathcal{G}} \boldsymbol{f}^{*} \cdot(\varepsilon \boldsymbol{f}) \mathrm{d} V\right| \leq \beta \iiint_{\mathcal{G}} \boldsymbol{f} \cdot \boldsymbol{f}^{*} \mathrm{~d} V$,

for some $0<\alpha, \beta<\infty$. The former part of the inequality is known as coerciveness [13], whereas the latter part of the inequality is known as boundedness.

As a result of the above conditions, $\varepsilon(\boldsymbol{r}, s)$ is invertible almost everywhere in space for a fixed value of $s$. Further, the modulus of every element of $\varepsilon(\boldsymbol{r}, s)$ is bounded from above, i.e., $\sup _{k, \ell, \boldsymbol{r} \in \overline{\mathcal{G}}}\left|\varepsilon_{k, \ell}(\boldsymbol{r}, s)\right|=\varepsilon_{\max }$. After splitting $\varepsilon(\boldsymbol{r}, s)$ in a real and imaginary part, i.e.,

$\varepsilon(\boldsymbol{r}, s)=\varepsilon(\boldsymbol{r}, s)^{\prime}-\mathrm{j} \varepsilon(\boldsymbol{r}, s)^{\prime \prime}$,

similar hypotheses on $\varepsilon(\boldsymbol{r}, s)$ exist, which can be derived from causality and finite-energy principles. These hypotheses are

$$
\begin{gathered}
0 \leq \boldsymbol{\xi}^{*} \cdot\left[\varepsilon(\boldsymbol{r}, s)^{\prime} \boldsymbol{\xi}\right] \leq a^{\prime} \boldsymbol{\xi}^{*} \cdot \boldsymbol{\xi}, \\
0 \leq \boldsymbol{\xi}^{*} \cdot\left[\varepsilon(\boldsymbol{r}, s)^{\prime \prime} \boldsymbol{\xi}\right] \leq a^{\prime \prime} \boldsymbol{\xi}^{*} \cdot \boldsymbol{\xi},
\end{gathered}
$$

for $\xi \in \mathbb{C}^{3}$ and where * denotes complex conjugation. The coefficients $a^{\prime}$ and $a^{\prime \prime}$ are independent of $\boldsymbol{r}$ and $s$. These hypotheses hold almost everywhere in $\mathcal{G}$, i.e., except for a set of volume zero, and indicate that both $\varepsilon^{\prime}$ and $\varepsilon^{\prime \prime}$ are positive semi-definite (almost everywhere). Furthermore, the conditions in (16) indicate that if the real part is indefinite in some region of $\mathcal{G}$, the imaginary part will be definite in that region, and vice versa, for a fixed $s$ (almost everywhere). A discussion of these hypotheses can be found in [14, Chap. 1]. 
Fig. 2 Extension of the domain $\mathcal{D}$ to $\mathcal{K}$

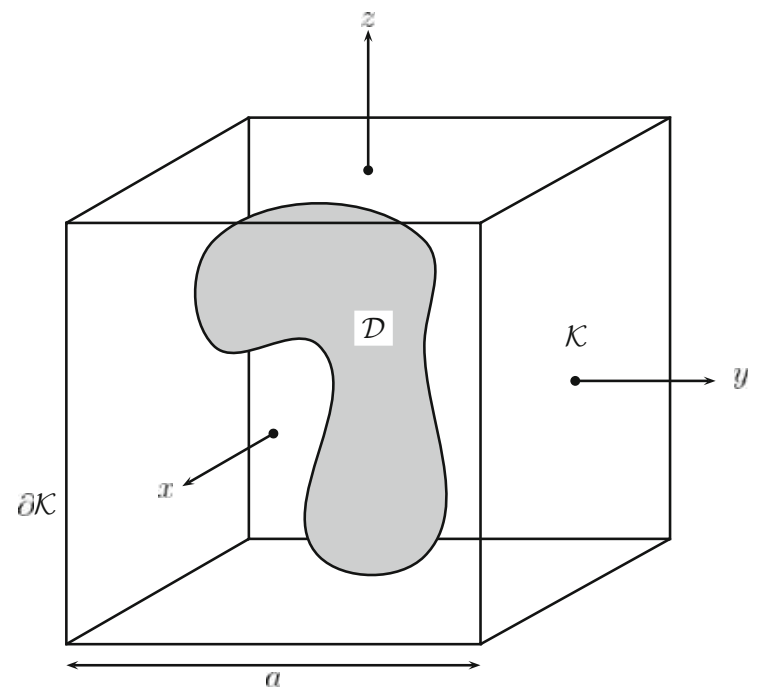

\subsection{Helmholtz decomposition}

To analyze the domain integro-differential equation, we extend the domain $\mathcal{D}$ to the cube $\mathcal{K}$, with boundary $\partial \mathcal{K}$, such that $\mathcal{D}$ is contained in $\mathcal{K}$ and the distance $d$ between $\mathcal{D}$ and $\partial \mathcal{K}$ is positive. This is shown in Fig. 2. We choose a Cartesian coordinate system such that its origin coincides with the center of the cube, the axes are oriented parallel to the edges of the cube and these edges have length $2 a$. Further, we extend the definition of the contrast-current density $\boldsymbol{J}^{c}$ to $\mathcal{K}$ by extending $\boldsymbol{J}^{c}$ by zero outside $\overline{\mathcal{D}}$.

Let $f$ be a continuously differentiable vector field on $\mathcal{K}$, i.e., $f \in C^{1}(\mathcal{K})^{3} \cap C(\overline{\mathcal{K}})^{3}$. Then the Helmholtz decomposition allows a decomposition of $\boldsymbol{f}$ as [15]

$\boldsymbol{f}=\nabla p+\nabla \times \boldsymbol{w}$,

where $p \in C^{2}(\mathcal{K})$ and $\boldsymbol{w} \in C^{1}(\mathcal{K})^{3} \cap C(\overline{\mathcal{K}})^{3}$. We can supplement the Helmholtz decomposition by a boundary condition for $w$

$\boldsymbol{n} \times\left.\boldsymbol{w}\right|_{\partial \mathcal{K}}=\mathbf{0}$,

where $\boldsymbol{n}$ is the outward-pointing unit normal vector at $\partial \mathcal{K}$. Using the identity

$\nabla \cdot(\boldsymbol{g} \times \boldsymbol{h})=\boldsymbol{h} \cdot \nabla \times \boldsymbol{g}-\boldsymbol{g} \cdot \nabla \times \boldsymbol{h}$,

where indicates the (non-complex) scalar product between two vectors, we have

$\iiint_{\mathcal{K}} \nabla \cdot\left(\boldsymbol{w}^{*} \times \nabla p\right) \mathrm{d} V=\iiint_{\mathcal{K}} \nabla p \cdot \nabla \times \boldsymbol{w}^{*} \mathrm{~d} V$,

where* indicates complex conjugation. From Gauss's theorem and Eq. (20), we obtain the orthogonality relation

$$
\iiint_{\mathcal{K}} \nabla p \cdot \nabla \times \boldsymbol{w}^{*} \mathrm{~d} V=\oiint_{\partial \mathcal{K}} \nabla p \cdot\left(\boldsymbol{n} \times \boldsymbol{w}^{*}\right) \mathrm{d} A=0 .
$$

Further, it can be proven [16, pp. 245-248 and 314] that the scalar field

$s(\boldsymbol{r})=\iiint_{\mathcal{K}} G\left(\boldsymbol{r}-\boldsymbol{r}^{\prime}\right) p\left(\boldsymbol{r}^{\prime}\right) \mathrm{d} V^{\prime}$,

satisfies the inhomogeneous Helmholtz equation

$\nabla^{2} s(\boldsymbol{r})-\frac{s^{2}}{c_{0}^{2}} s(\boldsymbol{r})=-p(\boldsymbol{r}), \quad \boldsymbol{r} \in \mathcal{K}$, 
where

$G(\boldsymbol{r})=\frac{\exp \left(-s|\boldsymbol{r}| / c_{0}\right)}{4 \pi|\boldsymbol{r}|}$.

For a continuously differentiable scalar function $q(\boldsymbol{r})$, we can show by straightforward integration by parts and by using the translation invariance of $G\left(\boldsymbol{r}-\boldsymbol{r}^{\prime}\right)$ that

$$
\iiint_{\mathcal{K}} G\left(\boldsymbol{r}-\boldsymbol{r}^{\prime}\right) \partial_{i^{\prime}} q\left(\boldsymbol{r}^{\prime}\right) \mathrm{d} V^{\prime}=\partial_{i} \iiint_{\mathcal{K}} G\left(\boldsymbol{r}-\boldsymbol{r}^{\prime}\right) q\left(\boldsymbol{r}^{\prime}\right) \mathrm{d} V^{\prime}+\iint_{\left.\partial \mathcal{K}\right|_{i=a}} G\left(\boldsymbol{r}-\boldsymbol{r}^{\prime}\right) q\left(\boldsymbol{r}^{\prime}\right) \mathrm{d} A^{\prime}-\iint_{\left.\partial \mathcal{K}\right|_{i=-a}} G\left(\boldsymbol{r}-\boldsymbol{r}^{\prime}\right) q\left(\boldsymbol{r}^{\prime}\right) \mathrm{d} A^{\prime},
$$

where $i \in\{x, y, z\}, i^{\prime} \in\left\{x^{\prime}, y^{\prime}, z^{\prime}\right\},\left.\partial \mathcal{K}\right|_{i=a}$ is the surface of the cube described by $i=a$ and $\left.\partial \mathcal{K}\right|_{i=-a}$ is the surface of the cube described by $i=-a$, e.g., $\left.\partial \mathcal{K}\right|_{x=a}$ is given by $x=a,-a<y<a,-a<z<a$. This identity, applied to the separate components in the Helmholtz decomposition, together with Eq. (20), yields

$$
\begin{aligned}
& \iiint_{\mathcal{K}} G\left(\boldsymbol{r}-\boldsymbol{r}^{\prime}\right) \nabla^{\prime} \times \boldsymbol{w}\left(\boldsymbol{r}^{\prime}\right) d V^{\prime}=\nabla \times \iiint_{\mathcal{K}} G\left(\boldsymbol{r}-\boldsymbol{r}^{\prime}\right) \boldsymbol{w}\left(\boldsymbol{r}^{\prime}\right) \mathrm{d} V^{\prime} \\
& \iiint_{\mathcal{K}} G\left(\boldsymbol{r}-\boldsymbol{r}^{\prime}\right) \nabla^{\prime} p\left(\boldsymbol{r}^{\prime}\right) \mathrm{d} V^{\prime}=\nabla \iiint_{\mathcal{K}} G\left(\boldsymbol{r}-\boldsymbol{r}^{\prime}\right) p\left(\boldsymbol{r}^{\prime}\right) \mathrm{d} V^{\prime}+\oiint_{\partial \mathcal{K}} G\left(\boldsymbol{r}-\boldsymbol{r}^{\prime}\right) p\left(\boldsymbol{r}^{\prime}\right) \boldsymbol{n}\left(\boldsymbol{r}^{\prime}\right) \mathrm{d} A^{\prime}
\end{aligned}
$$

The term containing the volume integral over $p$ is identical to $\nabla s(\boldsymbol{r})$ in Eq. (24). From Eqs. (7a) and (8), we observe that the scattered electric field is of the form

$s \varepsilon_{0} \boldsymbol{E}^{s}(\boldsymbol{r})=\left(\nabla \nabla \cdot-\frac{s^{2}}{c_{0}^{2}}\right) \iiint_{\mathcal{K}} G\left(\boldsymbol{r}-\boldsymbol{r}^{\prime}\right) \boldsymbol{f}\left(\boldsymbol{r}^{\prime}\right) \mathrm{d} V^{\prime}$,

where $\boldsymbol{f}(\boldsymbol{r})$ represents the contrast-current density $\boldsymbol{J}^{c}(\boldsymbol{r})$, after extension to $\mathcal{K}$. If we apply the results of Eqs. (19), (24), (25), and (28) to the representation of the scattered electric field, we obtain

$$
\begin{aligned}
& \left(\nabla \nabla \cdot-\frac{s^{2}}{c_{0}^{2}}\right) \iiint_{\mathcal{K}} G\left(\boldsymbol{r}-\boldsymbol{r}^{\prime}\right) \boldsymbol{f}\left(\boldsymbol{r}^{\prime}\right) \mathrm{d} V^{\prime}=\left(\nabla \nabla \cdot-\frac{s^{2}}{c_{0}^{2}}\right) \oiint_{\partial \mathcal{K}} G\left(\boldsymbol{r}-\boldsymbol{r}^{\prime}\right) p\left(\boldsymbol{r}^{\prime}\right) \boldsymbol{n}\left(\boldsymbol{r}^{\prime}\right) \mathrm{d} A^{\prime} \\
& -\nabla p-\frac{s^{2}}{c_{0}^{2}} \iiint_{\mathcal{K}} G\left(\boldsymbol{r}-\boldsymbol{r}^{\prime}\right) \nabla \times \boldsymbol{w}\left(\boldsymbol{r}^{\prime}\right) \mathrm{d} V^{\prime}
\end{aligned}
$$

for $\boldsymbol{r} \in \mathcal{K}$.

\subsection{Mapping properties under finite-energy conditions}

In view of the radiation conditions discussed in Sect. 3.2, we expect $\boldsymbol{J}^{c}$ to belong to the space $L^{2}(\mathcal{K})^{3}$, provided $\mathrm{M}_{\chi} \boldsymbol{E}^{i}$ also belongs to $L^{2}(\mathcal{K})^{3}$. Therefore, it is useful to investigate the mapping properties of the integro-differential operator I+ $\mathrm{M}_{\chi} \mathrm{N}$ as defined in Eq. (14c) as a mapping from $L^{2}(\mathcal{K})^{3}$ to $L^{2}(\mathcal{K})^{3}$. Because continuously differentiable functions are dense in $L^{2}(\mathcal{K})$, the Helmholtz decomposition can be extended to vector fields in $L^{2}(\mathcal{K})^{3}$ in the sense of Eq. (19) and the boundary condition (20) [15, Corollary 6, p. 226]. Hence we can define the mutually orthogonal projection operators $\mathrm{P}$ and $\mathrm{Q}$ as

$\mathrm{P} \boldsymbol{f}=\nabla p, \quad \mathrm{Q} \boldsymbol{f}=(\mathrm{I}-\mathrm{P}) \boldsymbol{f}=\nabla \times \boldsymbol{w}$,

where $\mathrm{P}$ and $\mathrm{Q}$ are mappings from $L^{2}(\mathcal{K})^{3}$ to $L^{2}(\mathcal{K})^{3}$. Accordingly, we have $p \in H^{1}(\mathcal{K})$ and $\boldsymbol{w} \in H_{t 0}^{1}(\mathcal{K})^{3}$, where

$$
\begin{aligned}
H^{1}(\mathcal{K}) & =\left\{g \mid g \in L^{2}(\mathcal{K}), \nabla g \in L^{2}(\mathcal{K})^{3}\right\}, \\
H_{t 0}^{1}(\mathcal{K})^{3} & =\left\{\boldsymbol{g}\left|\boldsymbol{g} \in H^{1}(\mathcal{K})^{3}, \quad \boldsymbol{n} \times \boldsymbol{g}\right|_{\partial \mathcal{K}}=\mathbf{0}\right\} .
\end{aligned}
$$


The scalar field $p$ is uniquely determined up to a constant, and the vector field $\boldsymbol{w}$ is unique under the supplementary condition

$\oiint_{S} w \cdot n \mathrm{~d} A=0$

for each regular closed surface $S \in \overline{\mathcal{K}}$. We will assume that the remaining constant of $p$ has been fixed by demanding

$\iiint_{\mathcal{K}} p \mathrm{~d} V=0$.

Owing to the same density properties, the results of Eq. (30) can be generalized to $f \in L^{2}(\mathcal{K})^{3}$. To this end, we define the operators $K$ and $L$ as

$\mathrm{K} \boldsymbol{f}=\frac{s^{2}}{c_{0}^{2}} \iiint_{\mathcal{K}} G\left(\boldsymbol{r}-\boldsymbol{r}^{\prime}\right) \boldsymbol{f}\left(\boldsymbol{r}^{\prime}\right) \mathrm{d} V^{\prime}$,
$\mathrm{L} \boldsymbol{f}=\left(\frac{s^{2}}{c_{0}^{2}}-\nabla \nabla \cdot\right) \oiint_{\partial \mathcal{K}} p\left(\boldsymbol{r}^{\prime}\right) G\left(\boldsymbol{r}-\boldsymbol{r}^{\prime}\right) \boldsymbol{n}\left(\boldsymbol{r}^{\prime}\right) \mathrm{d} A^{\prime}$,

where $\mathrm{K}: L^{2}(\mathcal{K})^{3} \rightarrow H^{2}(\mathcal{K})^{3}$ and $\mathrm{L}: L^{2}(\mathcal{K})^{3} \rightarrow C^{\infty}(\mathcal{K})^{3}$. The operator $\mathrm{L}$ is unambiguously defined if $p$ has been fixed according to Eq. (34), since in that case $p$ is uniquely determined by $\boldsymbol{f}$. Using these definitions and the preceding results for the Helmholtz decomposition of Eq. (30), we can write, for $\boldsymbol{J}^{c} \in L^{2}(\mathcal{K})^{3}$,

$\mathrm{M}_{\chi} \boldsymbol{E}^{i}=\frac{1}{s \varepsilon_{0}}\left(\mathrm{I}+\mathrm{M}_{\chi} \mathrm{N}\right) \boldsymbol{J}^{c}=\frac{1}{s \varepsilon_{0}}\left(\mathrm{I}+\mathrm{M}_{\chi} \mathrm{P}+\mathrm{M}_{\chi} \mathrm{KQ}+\mathrm{M}_{\chi} \mathrm{L}\right) \boldsymbol{J}^{c}$.

For this combination of operators, the following properties hold.

- I $+\mathrm{M}_{\chi} \mathrm{P}$ has a bounded inverse. This will be proven in Sect. 4.4. In view of Theorem 1, we will identify the operator $T$ with $I+M_{\chi} P$ and the remaining part $M_{\chi} K Q+M_{\chi} L$ will be identified with the compact operator $C$.

- The Green's function $G$ in the operator $\mathrm{K}$ is square integrable on $\mathcal{K}$ and therefore $\mathrm{K}$ is a Hilbert-Schmidt operator. In particular, $\mathrm{K}$ is compact from $L^{2}(\mathcal{K})^{3}$ to $L^{2}(\mathcal{K})^{3}$ [17]. This implies that the product $\mathrm{M}_{\chi} \mathrm{KQ}$ is compact from $L^{2}(\mathcal{K})^{3}$ to $L^{2}(\mathcal{K})^{3}$, since both $\mathrm{M}_{\chi}$ and $\mathrm{Q}$ are bounded.

This leaves us with the operator product $\mathrm{M}_{\chi} \mathrm{L}$. We observe that the function $\chi(\boldsymbol{r})$ is identically zero for $\boldsymbol{r}$ outside $\overline{\mathcal{D}}$, and we introduce an auxiliary operator $\mathrm{M}_{\phi}$,

$\mathrm{M}_{\phi} \boldsymbol{f}(\boldsymbol{r})=\phi(\boldsymbol{r}) \boldsymbol{f}(\boldsymbol{r})$,

where $\phi(\boldsymbol{r})$ is a $C_{0}^{\infty}(\mathcal{K})$ function such that

$$
\begin{cases}\phi(\boldsymbol{r})=1 & \text { for } \inf _{\boldsymbol{x} \in \partial \mathcal{K}}|\boldsymbol{x}-\boldsymbol{r}| \geq 2 d / 3, \\ 0 \leq \phi(\boldsymbol{r}) \leq 1 & \text { for } d / 3<\inf _{\boldsymbol{x} \in \partial \mathcal{K}}|\boldsymbol{x}-\boldsymbol{r}|<2 d / 3, \\ \phi(\boldsymbol{r})=0 & \text { for } \inf _{\boldsymbol{x} \in \partial \mathcal{K}}|\boldsymbol{x}-\boldsymbol{r}| \leq d / 3,\end{cases}
$$

for $\boldsymbol{r} \in \mathcal{K}$, and $d$ is the minimum distance between the boundary $\partial \mathcal{K}$ and the domain $\mathcal{D}$. We can now write

$\chi(\boldsymbol{r})=\chi(\boldsymbol{r}) \phi(\boldsymbol{r})+\chi(\boldsymbol{r})(1-\phi(\boldsymbol{r}))=\chi(\boldsymbol{r}) \phi(\boldsymbol{r})$,

owing to the fact that $\chi(\boldsymbol{r})=0$ outside the domain of the dielectric object. Hence,

$\mathrm{M}_{\chi}=\mathrm{M}_{\chi \phi}+\mathrm{M}_{\chi(1-\phi)}=\mathrm{M}_{\chi \phi}=\mathrm{M}_{\chi} \mathrm{M}_{\phi}$,

$\Rightarrow \mathrm{M}_{\chi} \mathrm{L}=\mathrm{M}_{\chi} \mathrm{M}_{\phi} \mathrm{L}$. 
We will prove that $\mathrm{M}_{\phi} \mathrm{L}$ is compact. The operator $\mathrm{M}_{\phi} \mathrm{L}$ is a bounded mapping from $L^{2}(\mathcal{K})^{3}$ to $C_{0}^{\infty}(\mathcal{K})^{3}$, since

$$
\sup _{\boldsymbol{r}^{\prime} \in \partial \mathcal{K}, \boldsymbol{r} \in \operatorname{supp}(\phi)}\left|\partial^{2} G\left(\boldsymbol{r}-\boldsymbol{r}^{\prime}\right)\right|<\infty,
$$

where $\partial^{2}$ denotes any second-order spatial derivative. This follows from the fact that the function $G$ is a $C^{\infty}$ function outside its singularity. The singularity is confined to $\partial \mathcal{K}$, since the integration is restricted to $\partial \mathcal{K}$. Further, $C_{0}^{\infty}(\mathcal{K})^{3}$ is a subspace of $H^{1}(\mathcal{K})^{3}$. Due to the compact-embedding theorem for Sobolev spaces [18, p. 144], $\mathrm{M}_{\phi} \mathrm{L}$ is a compact operator from $L^{2}(\mathcal{K})^{3}$ to $L^{2}(\mathcal{K})^{3}$. Finally, the operator $\mathrm{M}_{\chi}$ is bounded, as $\chi(\boldsymbol{r})$ is bounded. Since the product of a bounded operator and a compact operator is again compact, the operator $M_{\chi} M_{\phi} L$ is compact from $L^{2}(\mathcal{K})^{3}$ to $L^{2}(\mathcal{K})^{3}$. We conclude that $\mathrm{M}_{\chi} \mathrm{L}$ is a compact operator. It is for this reason that we have extended the domain $\mathcal{D}$ to the strictly larger cube $\mathcal{K}$. Moreover, the cube allows for an elementary Helmholtz decomposition since it is connected and simply connected. ${ }^{1}$

We end up with an operator splitting in the form as in Theorem 1, i.e., I $+M_{\chi} N=T+C$ with

$$
\mathrm{T}=\mathrm{I}+\mathrm{M}_{\chi} \mathrm{P}, \quad \mathrm{C}=\mathrm{M}_{\chi} \mathrm{KQ}+\mathrm{M}_{\chi} \mathrm{L} .
$$

Hence it remains to show that the operator $\mathrm{T}$ is bounded and has a bounded inverse, which will be proven in the next section.

\subsection{The operator I $+\mathrm{M}_{\chi} \mathrm{P}$}

The boundedness of $\mathrm{T}$ is a simple consequence of the boundedness of $\varepsilon(\boldsymbol{r}, s)$. To prove the existence and boundedness of the inverse, we decompose the operator $T$ in the form of a $2 \times 2$ operator matrix. This is possible, since $P$ and $\mathrm{Q}$ are mutually orthogonal projection operators and $\mathcal{R}(\mathrm{P}) \cup \mathcal{R}(\mathrm{Q})=L^{2}(\mathcal{K})^{3}$, where $\mathcal{R}(\mathrm{P})$ denotes the range of $P$.

$T=\left[\begin{array}{ll}P+P_{\chi} P & 0 \\ Q_{\chi} P & Q\end{array}\right]=\left[\begin{array}{ll}\mathrm{PM}_{I+\chi} P & 0 \\ \mathrm{QM}_{\chi} \mathrm{P} & \mathrm{Q}\end{array}\right]$,

where we have used the fact that $I+M_{\chi}=M_{I+\chi}$ and that $P$ is idempotent, since it is a projection operator. Hence, we can denote the mapping properties of $\mathrm{T}$ as

$\mathrm{T}:\left(\begin{array}{l}\mathcal{R}(\mathrm{P}) \\ \mathcal{R}(\mathrm{Q})\end{array}\right) \rightarrow\left(\begin{array}{c}\mathcal{R}(\mathrm{P}) \\ \mathcal{R}(\mathrm{Q})\end{array}\right)$

Further, $\mathrm{P}$ is equal to the identity operator on $\mathcal{R}(\mathrm{P})$ and $\mathrm{Q}$ is equal to the identity operator on $\mathcal{R}(\mathrm{Q})$. Therefore, straightforward linear algebra shows that we can construct an inverse of the form

$\mathrm{T}^{-1}=\left[\begin{array}{ll}\mathrm{PAP} & 0 \\ -\mathrm{QM}_{\chi} \mathrm{PAP} & \mathrm{Q}\end{array}\right]$,

where PAP should be the inverse of $\mathrm{PM}_{I+\chi} \mathrm{P}$ on $\mathcal{R}(\mathrm{P})$, which is not the same as $\mathrm{A}=\mathrm{M}_{I+\chi}^{-1}$. At the same time, if PAP does not exist, then the inverse of T does not exist. Therefore, a necessary and sufficient condition for the existence of the inverse of $\mathrm{I}+\mathrm{M}_{\chi} \mathrm{P}$ is that PAP exists. However, the operator $\mathrm{M}_{I+\chi}$ is coercive, as can be seen from the hypotheses on $\varepsilon$ in Eq. (16), since $I+\chi=\varepsilon / \varepsilon_{0}$. Further, the range of $\mathrm{P}$ is a Hilbert space with respect to the $L^{2}(\mathcal{K})^{3}$ inner product, and the coercivity of $\mathrm{PM}_{I+\chi} \mathrm{P}$ on $\mathcal{R}(\mathrm{P})$ is a direct consequence of the coerciveness of $\mathrm{M}_{I+\chi}$, i.e., $\mathrm{PM}_{I+\chi} \mathrm{P}$ is a bounded coercive linear operator on $\mathcal{R}(\mathrm{P})$. The Lax-Milgram theorem [13] guarantees the existence and boundedness of the inverse of $\mathrm{PM}_{I+\chi} \mathrm{P}$ on $\mathcal{R}(\mathrm{P})$, which is denoted as PAP.

\footnotetext{
${ }^{1}$ From the point of view of numerical methods, the cube is also a logical choice, since it allows straightforward meshing and the application of algorithms such as the Fast Fourier Transformation.
} 


\section{Integro-differential equations for $E$ and $D$}

From Sects. 3 and 4.3, it is clear that the integro-differential operators for $\boldsymbol{E}$ and $\boldsymbol{D}$, as defined in Eqs. (14a) and (14b), have an inverse which can be constructed from the bounded inverse of the integro-differential operator for the contrast current density $\boldsymbol{J}^{c}$. The main differences between the operators for $\boldsymbol{E}$ and $\boldsymbol{D}$, and the operator for $\boldsymbol{J}^{c}$, is that the order of the operators $\mathrm{M}_{\chi}$ and $\mathrm{N}$ is reversed. In that case, the existence and boundedness of the inverses is a consequence of the following more general algebraic result.

Lemma 1 Let $\mathrm{A}$ be a bounded operator from a Hilbert space $H_{1}$ to a Hilbert space $H_{2}$ and let $\mathrm{B}$ be a bounded operator from $\mathrm{H}_{2}$ to $\mathrm{H}_{1}$. The inverse of the operator $\mathrm{I}+\mathrm{AB}$ on $\mathrm{H}_{2}$ exist, if and only if the inverse of $\mathrm{I}+\mathrm{BA}$ on $\mathrm{H}_{1}$ exists. If the inverse of $\mathrm{I}+\mathrm{AB}$ exists, then the inverse of $\mathrm{I}+\mathrm{BA}$ can be constructed as $(\mathrm{I}+\mathrm{BA})^{-1}=\mathrm{I}-\mathrm{B}(\mathrm{I}+\mathrm{AB})^{-1} \mathrm{~A}$.

The proof follows by working out the algebra. This lemma can also be used to show that spectrum of the operator $A B$ is the same as the spectrum of BA, except possibly for the value 0, see e.g. [19, p. 196, Exerc. 8].

The range of $\mathrm{M}_{\chi}$ and the domain of $\mathrm{N}$ are clearly associated with $L^{2}(\mathcal{K})^{3}$, as a result of the analysis in Sect. 4.3. However, the domain of $\mathrm{M}_{\chi}$ and the range of $\mathrm{N}$ are not yet clear from that discussion. The range of $\mathrm{N}$ can be found via the Helmholtz decomposition on its domain space and then split $\mathrm{N}$ as in Sect. 4.3. In that way, we find that the range of $\mathrm{N}$ is contained in

$H_{\mathrm{loc}}\left(\operatorname{curl}, \mathbb{R}^{3}\right)=\left\{\boldsymbol{f} \mid \boldsymbol{f} \in L_{\mathrm{loc}}^{2}\left(\mathbb{R}^{3}\right)^{3} \wedge \nabla \times \boldsymbol{f} \in L_{\mathrm{loc}}^{2}\left(\mathbb{R}^{3}\right)^{3}\right\}$,

where the domain is $L^{2}(\mathcal{K})^{3} . L_{\text {loc }}^{2}\left(\mathbb{R}^{3}\right)^{3}$ is the space of three-dimensional vector functions which are locally square integrable. This aspect of the range of $\mathrm{N}$ is consistent with the observation that when $\mathrm{N}$ is applied to a contrast current density $\boldsymbol{J}^{c} \in L^{2}(\mathcal{K})^{3}$, then the result is an electric field, which satisfies Maxwell's curl equations. Further, since $H_{\text {loc }}\left(\right.$ curl, $\mathbb{R}^{3}$ ) is a subspace of $L_{\text {loc }}^{2}\left(\mathbb{R}^{3}\right)^{3}, \mathrm{M}_{\chi} \mathrm{N}$ maps $L^{2}(\mathcal{K})^{3}$ into $L^{2}(\mathcal{D})^{3} \subset L^{2}(\mathcal{K})^{3}$ (cf. Sect. 4.1).

If we choose to employ the domain integro-differential equation (14b) for the generalized electric-flux density, then we also need to consider proper Hilbert spaces for the domain and range of $M_{\varepsilon^{-1}}$. Since $M_{\varepsilon^{-1}}$ maps the electric-flux density to an electric field, the proper space for the range of $M_{\varepsilon^{-1}}$ would again be $H_{\text {loc }}\left(\operatorname{curl}, \mathbb{R}^{3}\right.$ ). For the domain, we recall that the electric-flux density satisfies a divergence equation in Maxwell's equations. Therefore, a suitable Hilbert space for the domain of $\mathrm{M}_{\varepsilon^{-1}}$ is

$H_{\mathrm{loc}}\left(\operatorname{div}, \mathbb{R}^{3}\right)=\left\{\boldsymbol{f} \mid \boldsymbol{f} \in L_{\mathrm{loc}}^{2}\left(\mathbb{R}^{3}\right)^{3} \wedge \nabla \cdot \boldsymbol{f} \in L_{\mathrm{loc}}^{2}\left(\mathbb{R}^{3}\right)\right\}$.

Hence, if the permittivity operator $\mathrm{M}_{\varepsilon}$ is an invertible mapping from $H_{\mathrm{loc}}\left(\operatorname{curl}, \mathbb{R}^{3}\right)$ to $H_{\mathrm{loc}}\left(\operatorname{div}, \mathbb{R}^{3}\right)$, which is explicitly required for the construction of the integro-differential equation, then the analysis reveals that the operator $M_{\varepsilon^{-1}}$ in Eq. (14b) defines a mapping from $H_{\text {loc }}\left(\operatorname{div}, \mathbb{R}^{3}\right)$ to $H_{\text {loc }}\left(\operatorname{curl}, \mathbb{R}^{3}\right)$ and it has a bounded inverse.

The use of the Sobolev spaces $H_{\text {loc }}\left(\operatorname{curl}, \mathbb{R}^{3}\right)$ and $H_{\text {loc }}\left(\operatorname{div}, \mathbb{R}^{3}\right)$ do not invite us to develop a practical numerical scheme. Thus we would like to consider Eq. (14a) on a restricted domain rather than the full $\mathbb{R}^{3}$, on which the total electric field $\boldsymbol{E}$ is to be approximated, since otherwise we would have to find an approximation on all $\mathbb{R}^{3}$. We will not consider the most general restrictions of the domain. Instead we will look at the restriction to a cube $\mathcal{K}_{1}$, which contains $\overline{\mathcal{D}}$ and which is strictly smaller than the cube $\mathcal{K}$, on which the Helmholtz decomposition in Sect. 4 was performed. We define $R_{\mathcal{K}_{1}}$ as the restriction operator with respect to the cube $\mathcal{K}_{1}$. The operator $\mathrm{R}_{\mathcal{K}_{1}}$ has three important properties. First, on $\mathcal{K}_{1}$ it is equal to the identity operator. Second, $\mathrm{R}_{\mathcal{K}_{1}}$ is idempotent, and third,

$\mathrm{M}_{\chi}=\mathrm{R}_{\mathcal{K}_{1}} \mathrm{M}_{\chi}=\mathrm{M}_{\chi} \mathrm{R}_{\mathcal{K}_{1}}$.

These properties together with Lemma 1, allow us to construct the inverse of the restricted operator $R_{\mathcal{K}_{1}}\left(I+N M_{\chi}\right) R_{\mathcal{K}_{1}}$ for the restricted total electric field $R_{\mathcal{K}_{1}} \boldsymbol{E}$, in terms of the inverse of the operator $I+M_{\chi} N$ for the contrast current density on $\mathcal{K}$. The inverse of $\mathrm{R}_{\mathcal{K}_{1}}\left(\mathrm{I}+\mathrm{NM}_{\chi}\right) \mathrm{R}_{\mathcal{K}_{1}}$ on $\mathcal{K}_{1}$ is now given by

$\left[\mathrm{R}_{\mathcal{K}_{1}}\left(\mathrm{I}+\mathrm{NM}_{\chi}\right) \mathrm{R}_{\mathcal{K}_{1}}\right]^{-1}=\mathrm{R}_{\mathcal{K}_{1}}\left(\mathrm{I}-\mathrm{NUM}_{\chi}\right) \mathrm{R}_{\mathcal{K}_{1}}$,

where $U$ is the inverse of $I+M_{\chi} N$ restricted to $\mathcal{K}_{1}$, i.e.,

$\mathrm{U}=\mathrm{R}_{\mathcal{K}_{1}}\left(\mathrm{I}+\mathrm{M}_{\chi} \mathrm{N}\right)^{-1} \mathrm{R}_{\mathcal{K}_{1}}$, 
which exists on $\mathcal{K}_{1}$ since $\mathcal{K}$ is strictly larger than $\mathcal{K}_{1}$. The proof follows by working out the algebra and by taking into account the properties of $R_{\mathcal{K}_{1}}$ mentioned above. Hence the domain of the integro-differential equation for the electric field (Eq. (14a)) can be restricted to the cube $\mathcal{K}_{1}$, without affecting the existence and boundedness of its inverse. The same argument holds for Eq. (14b). The domain and range of the operator $R_{\mathcal{K}_{1}}\left(I+N M_{\chi}\right) R_{\mathcal{K}_{1}}$ is then equal to $H$ (curl, $\mathcal{K}_{1}$ ), which is defined as [15]

$H\left(\operatorname{curl}, \mathcal{K}_{1}\right)=\left\{\boldsymbol{f} \mid \boldsymbol{f} \in L^{2}\left(\mathcal{K}_{1}\right)^{3} \wedge \nabla \times f \in L^{2}\left(\mathcal{K}_{1}\right)^{3}\right\}$.

For the restricted electric flux density, the proper Hilbert space is [15]

$H\left(\operatorname{div}, \mathcal{K}_{1}\right)=\left\{\boldsymbol{f} \mid \boldsymbol{f} \in L^{2}\left(\mathcal{K}_{1}\right)^{3} \wedge \nabla \cdot \boldsymbol{f} \in L^{2}\left(\mathcal{K}_{1}\right)\right\}$.

It is also possible to prove the existence and boundedness of the inverse of $R_{\mathcal{K}_{1}}\left(I+N M_{\chi}\right) R_{\mathcal{K}_{1}}$ on $\mathcal{K}_{1}$ directly, by employing the Helmholtz decomposition on the cube $\mathcal{K}$, as in Sect. 4. Then, we obtain

$\mathrm{R}_{\mathcal{K}_{1}}\left(\mathrm{I}+\mathrm{NM}_{\chi}\right) \mathrm{R}_{\mathcal{K}_{1}}=\mathrm{R}_{\mathcal{K}_{1}}+\mathrm{R}_{\mathcal{K}_{1}} \mathrm{PM}_{\chi}+\mathrm{R}_{\mathcal{K}_{1}} \mathrm{KQM}_{\chi}+\mathrm{R}_{\mathcal{K}_{1}} \mathrm{LM}_{\chi}$.

The latter two operator combinations are again compact from $H\left(\right.$ curl, $\left.\mathcal{K}_{1}\right)$ to $H$ (curl, $\mathcal{K}_{1}$ ) by the same arguments as in Sect. 4.3, owing to the fact that $\mathcal{K}_{1}$ is strictly smaller than $\mathcal{K}$. Hence, we observe that the operator $\mathrm{R}_{\mathcal{K}_{1}}\left(\mathrm{I}+\mathrm{PM} \mathrm{M}_{\chi}\right) \mathrm{R}_{\mathcal{K}_{1}}$ should have a bounded inverse on $H\left(\operatorname{curl}, \mathcal{K}_{1}\right)$. This is again established via Lemma 1 and the conditions on the permittivity function.

\section{Numerical consequences}

From the preceding analysis it is clear that the domain integro-differential equation for the contrast-current density has a bounded inverse from $L^{2}(\mathcal{K})^{3}$ to $L^{2}(\mathcal{K})^{3}$. In other words, the problem is well-posed from $L^{2}(\mathcal{K})^{3}$ to $L^{2}(\mathcal{K})^{3}$. Similar observations have been made for the domain integro-differential equations for the electric field and for the generalized electric-flux density, albeit for different functions spaces. For a projection method, this means that, in principle, we can adopt the Galerkin method with respect to the $L^{2}(\mathcal{K})^{3}$ inner product and with basis and testing functions belonging to $L^{2}(\mathcal{K})^{3}$. Alternatively, one could construct basis and testing functions for the Helmholtz decomposition. However, the presented analysis is not directly applicable in a practical numerical tool that employs local basis and testing functions.

The proof of well-posedness given here, based on the splitting of the integro-differential operator, also shows that the permittivity function plays a role in the dominant part of the integro-differential operator, i.e., T. However, its contribution is restricted to the laminar part. Hence, large or negative contrasts cause a large difference in scale between the laminar and the solenoidal parts of the operator $\mathrm{T}$, which has a negative effect on the conditioning of the entire integro-differential operator and its discretized counterpart. This explains why iterative techniques tend to stall in their convergence for such cases. Further, it indicates that a suitable preconditioner should be based on an approximate inverse of the operator $T$ or its discretized counterpart.

At present, numerical schemes based on Eq. (10) [20] and Eq. (11) [1-3,21] are available. In these schemes, which employ the $L^{2}(\mathcal{D})^{3}$ inner product, the term $\nabla \nabla \cdot \boldsymbol{A}$ is tackled by bringing the gradient operator over to the testing function, which is piecewise linear in the longitudinal direction. As a result, a finite-difference formula is obtained for the differential operator. For the formulation where the Green's function has been replaced by a spatially averaged version of the Green's function [1-3,20], this approximation is valid, since the weak Green's function is continuous everywhere. It has been demonstrated that the weak Green's function converges to the original Green's function when the radius of the sphere, on which the Green's function is averaged, goes to zero. However, further research is required to examine whether this also holds for the combination of the integro-differential equation and the weak Green's function. For all of the methods mentioned above, it is not clear that the dual space of the range space of the domain integro-differential operator can be spanned by the proposed testing functions, since the differentiability of these testing functions is used explicitly. This results in a solution space for the contrast-current density that is larger than $L^{2}(\mathcal{K})^{3}$. In turn, this larger space may no longer give rise to a unique solution of the 
domain integro-differential equations. Therefore, we cannot guarantee convergence in norm for these methods. These existing discretization schemes can be improved by employing less smooth testing functions, e.g. piecewise constant functions. However, it is not a priori clear that the resulting discretized operator exhibits convergence in norm, since the dominant operator $\mathrm{T}$ is neither the identity operator nor a coercive one. Therefore, standard arguments for convergence do not apply here and a more detailed study is required. By virtue of [13, Theorem 13.7], such a study should focus on the invertibility and conditioning of the discretized version of $T$ with respect to mesh refinement. Finally, in [22], a "potential integral equation method" was proposed. The advantage of its formulation is that the second-order spatial derivatives operating on the vector potential $(\nabla \nabla \cdot \boldsymbol{A})$ have been removed, at the expense of spatial derivatives operating on the permittivity function. Therefore, this formulation is restricted to sufficiently smooth profiles. The numerical scheme proposed for this formulation is based on point-matching, which implies that convergence in an optimal norm cannot be expected.

\section{Conclusion}

Under general assumptions for the properties of a dielectric object, the domain integro-differential equations for the contrast current density, for the electric field and generalized electric-flux density both restricted to $\mathcal{K}_{1}$ have a bounded inverse from $L^{2}(\mathcal{K})^{3}$ to $L^{2}(\mathcal{K})^{3}$, from $H\left(\operatorname{curl}, \mathcal{K}_{1}\right)$ to $H$ (curl, $\left.\mathcal{K}_{1}\right)$, and from $H\left(\operatorname{div}, \mathcal{K}_{1}\right)$ to $H\left(\operatorname{curl}, \mathcal{K}_{1}\right)$, respectively. We have shown that these equations can be reduced to a Fredholm integral equation of the second kind with a unique solution. We have proven equivalence relations between the inverses of the three operators. The problems are well-posed for the above mentioned function spaces and the conditions for the well-posedness of the equations are identical.

Open Access This article is distributed under the terms of the Creative Commons Attribution Noncommercial License which permits any noncommercial use, distribution, and reproduction in any medium, provided the original author(s) and source are credited.

\section{References}

1. Zwamborn APM (1991) Scattering by objects with electric contrast. Dissertation, Delft University of Technology

2. Zwamborn APM, van den Berg PM (1992) The three-dimensional weak form of the conjugate gradient FFT method for solving scattering problems. IEEE Trans Microwave Theory Tech 40:1757-1766

3. Zwamborn APM, van den Berg PM (1994) Computation of electromagnetic fields inside strongly inhomogeneous objects by the weak conjugate gradient FFT method. JOSA A 11:1414-1421

4. Chew WC, Jin J, Michielssen E (eds) (2001) Fast and efficient algorithms in computational electromagnetics. Artech House, London

5. Müller C (1969) Foundations of the mathematical theory of electromagnetic waves. Springer-Verlag, Berlin

6. Pankratov OV, Avdeev DB, Kuvshinov AV (1995) Electromagnetic field scattering in a heterogeneous earth: a solution to the forward problem. Phys Solid Earth 31:201-209 (English edn)

7. Singer BS (1995) Method for solution of Maxwell's equations in non-uniform media. Geophys J Int 120(3):590-598

8. Singer BS, Fainberg EB (1995) Generalization of the iterative dissipative method for modeling electromagnetic fields in nonuniform media with displacement currents. Appl Geophys 34:41-46

9. Zhdanov MS, Fang S (1997) Quasi-linear series in three-dimensional electromagnetic modeling. Radio Sci 32:2167-2188

10. de Hoop AT (1995) Handbook of radiation and scattering of waves. Academic Press, London

11. Jones DS (1964) The theory of electromagnetism. Pergamon, Oxford

12. Colton D, Kress R (1983) Integral equations in scattering theory. Wiley, New York

13. Kress R (1989) Linear integral equations. Springer-Verlag, Berlin

14. Cessenat M (1996) Mathematical methods in electromagnetism, linear theory and applications. World Scientific, Singapore

15. Dautray R, Lions J-L (1990) Mathematical analysis and numerical methods for science and technology, vol 3. Springer-Verlag, Berlin

16. Courant R, Hilbert D (1989) Methods of mathematical physics, vol 2, Wiley Classics edition. Wiley, New York

17. Groetsch C (1980) Elements of applicable functional analysis. Marcel Dekker Inc, New York

18. Adams RA (1975) Sobolev spaces. Academic Press, London

19. Hirsch F, Lacombe G (1999) Elements of functional analysis, graduate texts in mathematics. Springer, New York

20. Abubakar A (2000) Three-dimensional nonlinear inversion of electrical conductivity. Dissertation, Delft University of Technology 
21. Schaubert DH, Wilton DR, Glisson AW (1984) A tetrahedral modeling method for electromagnetic scattering by arbitrarily shaped imhomogeneous dielectric bodies. IEEE Trans Antennas Propagat 32:77-85

22. De Donker P (2001) A potential integral equation method for electromagnetic scattering by penetrable bodies. IEEE Trans Antennas Propagat 49:1037-1042 